\title{
Development of an automated modern undergraduate optics laboratory using LabVIEW
}

\section{Amit Garg, Reena Sharma, Vishal Dhingra}

Amit Garg, Reena Sharma, Vishal Dhingra, "Development of an automated modern undergraduate optics laboratory using LabVIEW," Proc. SPIE 9666, 11th Education and Training in Optics and Photonics Conference, 966617 (5 June 2009); doi: 10.1117/12.2208037

SPIE Event: Eleventh International Topical Meeting on Education and Training in Optics and Photonics, 2009, St. Asaph, United Kingdom 


\title{
Development of an Automated Modern Undergraduate Optics Laboratory using LabVIEW
}

\author{
Amit Garg, Reena Sharma, Vishal Dhingra \\ Department of Electronics \\ Acharya Narendra Dev College, University of Delhi \\ Govindpuri, Kalkaji, New Delhi-110019, India
}

\begin{abstract}
We report here the development of an automated modern optics laboratory for undergraduate students. This developed modern optics laboratory have automated experiments on optoelectronic device characterisation, optical instrumentation, CCD based optical experiments and advanced applications in optics. In the device characterisation section, Voltage-Current $(\mathrm{V}-\mathrm{I})$ and Optical Power-Current $(\mathrm{P}-\mathrm{I})$ characteristics of various optoelectronic devices like LEDs, Laser diodes and photo diodes have been automated. In the optical instrumentation section, development of PC based optical power meter have been reported whose functionality can be tailored as per the need of the designed experiment. In the CCD based optical experiments section, CCD has been integrated for fringe capture and analysis by studying the diffraction pattern of a pinhole. In the advanced applications in optics section, molar absorbivity of $\mathrm{NiSO}_{4}$ has been calculated. Further work is in progress to develop heart rate monitoring system, non-evasive jaundice studies from the skin, CCD based real time spectrometers as well as elaborate studies on interference, diffraction and polarisation. The automation of this laboratory has been done by integrating various sensors (photodetectors, CCDs, Current and others) with data acquisition cards connected to PC and one of the most widely used world wide scientific graphical programming software LabVIEW. The purpose of the laboratory is to invoke student interest by exposing them to various modern tools in comparison to very conventional as well as boring existing optics laboratories. The use of this scientific graphical programming software will help in performing various real time measurements and calculations with ease. The automation of the experiments will also save great amount of experimentation time and procurement of costly equipments dedicated to each experiment thus providing an efficient way to carry out studies with reduced financial constraints and better manoeuvrability.
\end{abstract}

Keyword List: Modern Optics Laboratory, Computer Interfacing, Automation of Experiments

Email: amit_andc@yahoo.co.in, Ph. 911126412547, Fax 911126294540

Eleventh International Topical Meeting on Education and Training in Optics and

Photonics, edited by K. Alan Shore, Deb Kane, Proc. of SPIE Vol. 9666, 966617

(c) 2009 SPIE, OSA, IEEE, ICO · doi: 10.1117/12.2208037 


\section{INTRODUCTION}

Optics is a branch of physics that deals with light. It is related to a number of phenomena from the lovely sight of rainbow in the sky to the laser light in a laboratory. Applied optics is related to different applications of optics. Lasers and their applications have added new dimensions to the study of optics. Laser in its different incarnations such as a doctor's scalpel doing bloodless surgery, booming laser-guided missile in space, weed-killer in undulating crops and as a tool in the optics laboratory has made optics and applied optics the most feted frontier of science.

The need for enhanced undergraduate laboratory teaching aids has been identified for better understanding of basic concepts to students. Although there are currently a number of interesting and powerful educational products in the market, they are typically produced in the developed countries and are sometimes inappropriate for applications in the developing countries. They are also often very expensive. This paper describes an automated modern optics laboratory configuration and a set of tools designed and implemented at the undergraduate level. The laboratory encompasses a personal computer (PC) station and a set of inexpensive hardware tools for data acquisition and control to make learning for students innovative. ${ }^{[1],[2]}$

The optics laboratory consists of four sections: Optical Instrumentation, Opto-Electronic Device Characterization, CCD based Optical Experiments, and Advanced Applications in Optics. The four sections of this automated modern optics laboratory have been discussed as under:

\section{Section I: Optical Instrumentation}

Optical instrumentation has made the overall expansion of optics and applied optics feasible. Primary working

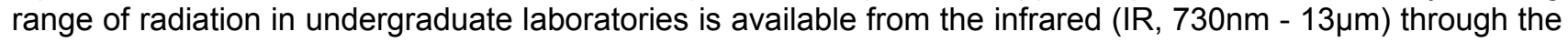
visible $(380 \mathrm{~nm}-780 \mathrm{~nm})$ region. So the instrumentation must respond to this range of radiation.

The developed optics laboratory comprises of an important instrument to measure optical power. An optical power meter is a device which converts light power to a measurable current or voltage that is proportional to the optical input. The optical power meter is used to monitor the power of the laser generating the optical signal, optics experiments based on various types of lasers in the undergraduate and postgraduate physics laboratory, to measure the loss through the transmission medium, to test the receiving electronics, optical communication industry, etc.

\section{Theory}

Optical power meters comprise of a photo-detector which generates an electrical current proportional to the optical power of an input optical beam, a Current to Voltage (I/V) amplifier (trans-impedance amplifier) which converts the output current of this photo-detector to a voltage, a variable gain amplifier which amplifies the output voltage of this I/V amplifier, and an A/D converter which converts the output voltage of this variable gain amplifier to a digital signal. Optical power meters generally do not discriminate wavelengths, rather they measure light intensity or optical power independently or substantially independently of wavelength.

In this paper, we report the development of a computer-based optical power meter which is a low-cost system containing a photo-detector and a USB based data acquisition card from National Instruments (NI-DAQ USB 6008). It offers all the features of standalone box type optical power meters in addition to scalability to higher number of channels, advanced data analysis, and continuous collection of optical power measurements.

\section{Experimental Set-up}

When interfacing with a photo-detector, the quantity that must be measured is current. A trans-impedance amplifier has been employed to measure the current as it is the most suitable method that yields the detectivity, signal-to-noise, and accuracy that is expected from a semiconductor photo-detector. The 
advantage that the trans-impedance amplifier has over almost any other amplifier configuration is that it does not bias the photodiode with a voltage as the current starts to flow from the photodiode. Typically, one lead of the photo-detector is tied to the ground and the other lead is kept at virtual ground by means of the input of the trans-impedance amplifier. The resultant bias across the photo-detector is then kept at virtually zero volts, a condition that helps minimize dark current and noise, and helps increase linearity and detectivity. Effectively the trans-impedance amplifier causes the photocurrent to flow through the feedback resistor, which creates a voltage, $V=i R$ ( $i$ is the current generated by photodiode and $R$ is the feedback resistor), at the output of the amplifier. Since the designed program knows the value of the precision feedback resistor, the current can be calculated with very good accuracy.

The experimental design of the optical power meter is as shown in Fig.1.

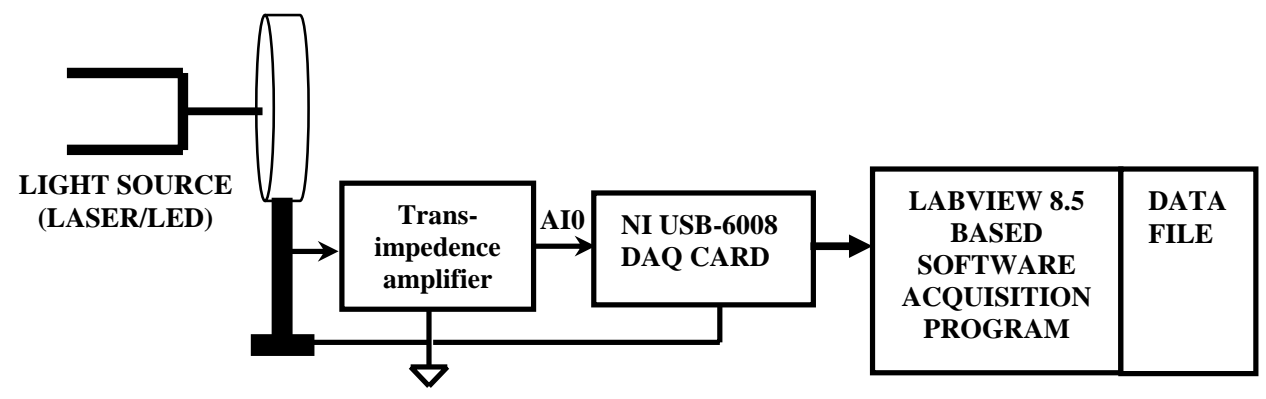

Fig. 1: Experimental Design of Optical Power Meter

The experimental set-up consists of two sections: Hardware and Software. The hardware section comprises of a photodetector, transimpedance amplifier and a NI USB-6008 DAQ Card as shown in Fig.1. The light from the source under study is incident on the photodetector which generates an output current associated with the detector responsivity. This current is then converted to an equivalent analog voltage using a transimpedance amplifier as explained above. The analog voltage is fed to the LabVIEW based software section using the DAQ Card. The developed setup also provides an advantage of using any detector in the laboratory without the manufacturer data.

The software section developed using Student Version of LabVIEW 8.5.1 works in two modes: Calibration and Measurement. Prior to any measurement of optical power, the photodetector in the system is calibrated for accurate power measurements. The calibration routine in the software measures/calculates the responsivity for the photodetector in use. Responsivity is expressed as current/optical power (A/W) and is usually provided by the manufacturer of the photodetector. However the developed software eliminates the need of the manufacturer data for calibration.

The calibration routine in the software incorporates this feature in 3 different modes: Single Wavelength Calibration, Multiple Wavelength Calibration and Standard Detector Calibration. The three modes have been described elsewhere. ${ }^{[3]}$

After the calibration of the detector is over, the software enables the options for optical power measurement. On clicking the button, "Measure Optical Power" on the software, the software asks the user to input the wavelength of the source for which optical power is to be measured. Using the results in the calibration section, the software now calculates the optical power for the source under study. At every click for power measurement, the software measures the optical power 100 times and displays them on a waveform chart in the front panel of the software. This data is useful to study the stability of optical detector and the source of light over time. The average value of the optical power is indicated by a numerical indicator on the panel. The software is also capable of displaying optical power in all the commonly used units such as Watts (W), 
milliwatts $(\mathrm{mW})$, microwatts $(\mu \mathrm{W})$, nanowatts $(\mathrm{nW})$, picowatts $(\mathrm{pW})$ and $\mathrm{dBm}$. The various screenshots and the supporting program are as shown in Fig.2 (a)-2(c).

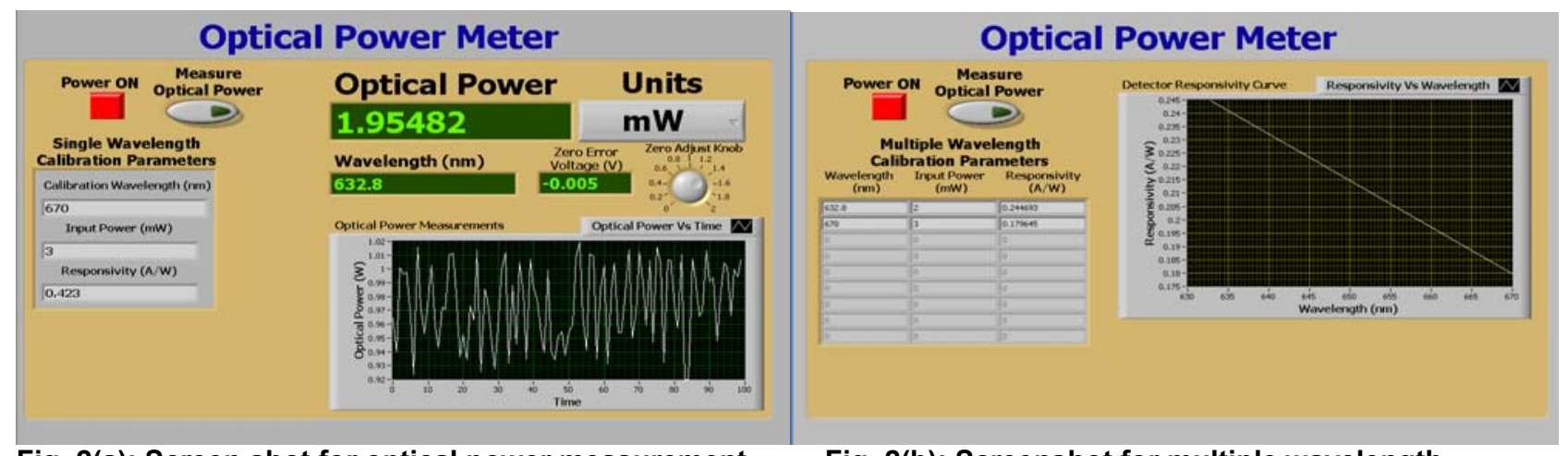

Fig. 2(a): Screen shot for optical power measurement using single wavelength calibration.
Fig. 2(b): Screenshot for multiple wavelength calibration.

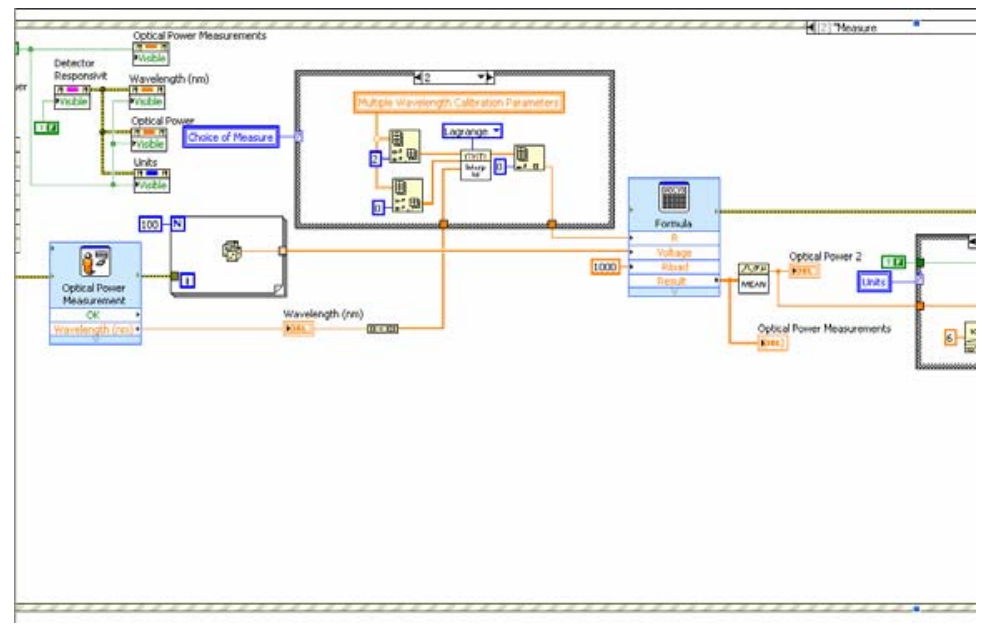

Fig. 2(c): Part of the developed software code in LabVIEW 8.5.1.

\section{Results}

The developed system has been checked using a standard He-Ne laser at $632.8 \mathrm{~nm}$ of known optical power output of $2 \mathrm{~mW}$. The responsivity of the photodetector used in the setup is $0.412 \mathrm{~A} / \mathrm{W}$. The standard He-Ne laser source output beam was directly positioned onto the photodetector. The developed system estimates the optical power output for the laser as $1.95482 \mathrm{~mW}$. Also, to check the linearity of the system, we put a $50 / 50$ beam splitter in the beam path of the He-Ne laser so as to reduce the output power falling onto the detector to half i.e. $1 \mathrm{~mW}$. The recorded value on the power meter is $0.97738 \mathrm{~mW}$. Hence the response of the system is linear.

In order to check the calibration at other wavelengths, we used a diode laser of $670 \mathrm{~nm}$ having an optical power output of $3 \mathrm{~mW}$. After calibration as per the datasheet of the photodetector, the power recorded on the optical power meter is $2.92 \mathrm{~mW}$ which is near the actual power.

Therefore, the developed optical power meter is quite reliable and accurate. One important feature of the system is that it shows the time based waveform recording besides the numerical value of the power. Therefore, output stability of the source over a period of time can be displayed. 


\section{SECTION II: Opto-Electronic Device Characterization}

Light Emitting Diodes (LEDs) and laser diodes are the most widely used optical sources for optical communication and relevant studies. A laser diode works in two modes, spontaneous and stimulated emission. However, a light emitting diode works on the principle of spontaneous emission only. Use of these devices for various applications involves correct biasing conditions. The regions of operation can be identified by studying the two most important characteristics, i.e., device current vs. device voltage (I-V) characteristics and optical output power vs. device current (P-I) characteristics.

We have developed an automated device characterization unit for study of various optical devices. This unit helps to study I-V characteristics for LEDs, Laser Diode, Photo detectors or any other optical device. The laser and LEDs being the light sources, the P-I characterization unit focuses on their study only. Several laser parameters and characteristics can be ascertained by study of its P-I curve. The device under test is just to be connected between the test points and all the measurements are done automatically by the developed system.

\section{Theory}

Like all diodes, both these diodes are inherently non-linear devices with respect to voltage and current. After applying a very small voltage, current flows easily through a diode without any significant increase in voltage. LEDs and Laser diodes operating in the forward region act in similar manner. However, they are unique because they emit a coherent optical beam as current is driven through the device. So the relationship between current and the light output is important.

At first, a significant amount of current seems to produce no light, and then the light output increases rapidly after a threshold point of current is reached. Below this point, any light emitted from the device is the diodes spontaneous emission. The population inversion is not great enough here to create stimulated emission, but still the holes and electrons are recombining. The emission is spontaneous, the light generated is low, and the light is incoherent and in all directions. Stimulated emission starts at the threshold current value. Determination of threshold current is especially important for modulated lasers. For efficient high-speed operation, diode lasers are biased around the threshold current for rapid switching response. At higher temperatures, threshold current increases to higher value and hence more current is needed to turn on the laser for a given amount of output optical power. ${ }^{[4],[5],[6]}$

\section{Experimental Set-up}

The automated characterization module was built using Student Version of LabVIEW 8.5.1 and its compatible Data Acquisition (DAQ) card, NI USB-6008 for interfacing. The experimental set-up and its working for the two different characteristic studies are explained as under:

1. I-V Characteristics: The block diagram for the I-V characteristic study is shown in Fig. 3.

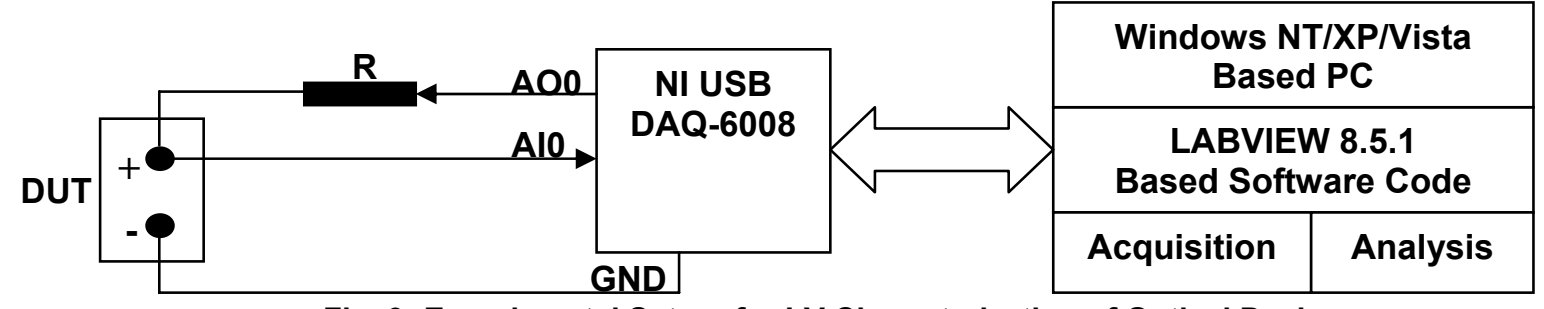

Fig. 3: Experimental Set-up for I-V Characterization of Optical Devices

The Device Under Test (DUT) is placed between the test points and the characteristic curve is acquired and plotted for the device by LabVIEW along with DAQ card which provides for all the parameter acquisition. 
Analog Output Channel 0 (AO0) of the DAQ card is used to supply voltage to the DUT in steps of 0.01 Volts and the corresponding device voltage is read using Analog Input Channel 0 (AIO). The difference in the two voltages is then used to calculate the current flowing through the series resistor, $R$ which is same as the device current in this case. This current is plotted with respect to the voltage read from AlO thus generating the I-V characteristic curve for the DUT. The I-V characteristic curve is fitted with the equation (1)

$$
l=A\left(e^{B V}-1\right)
$$

Equation (1) when compared with the diode equation (2) is used to determine important parameters such as reverse saturation current, $I_{0}$ and $\eta V_{T}$ where $\eta$ is the ideality factor and $V_{T}$ is the equivalent thermal voltage.

$$
r=I_{\mathrm{p}}\left(e^{\mathrm{V} / \mathrm{p} \mathrm{V}_{z}}-1\right)
$$

The device characteristic is simultaneously stored in a datalog file which can be analysed anytime later for further study. The results obtained from the I-V characteristics for an LED, a laser diode and a photodiode are shown in Fig. 4(a)-(c). Figure 5 shows a part of the graphical software code designed in LabVIEW.

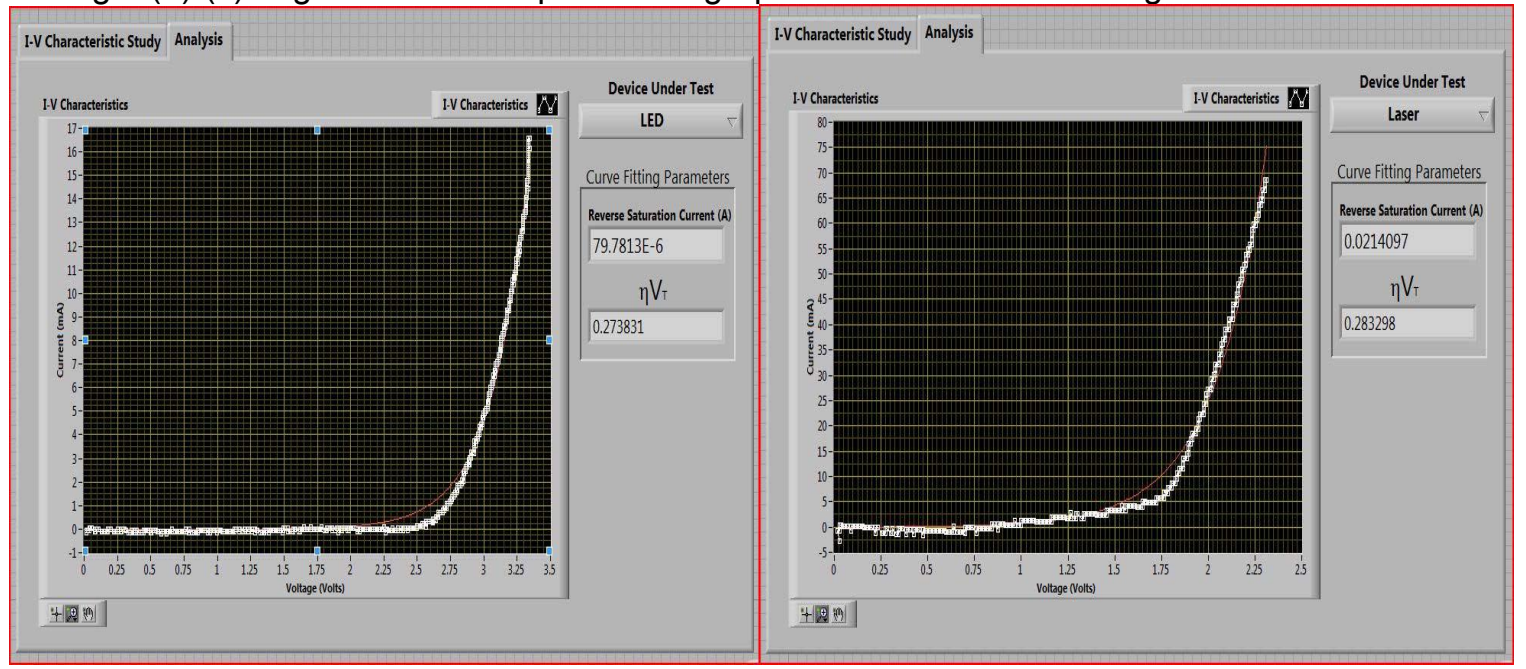

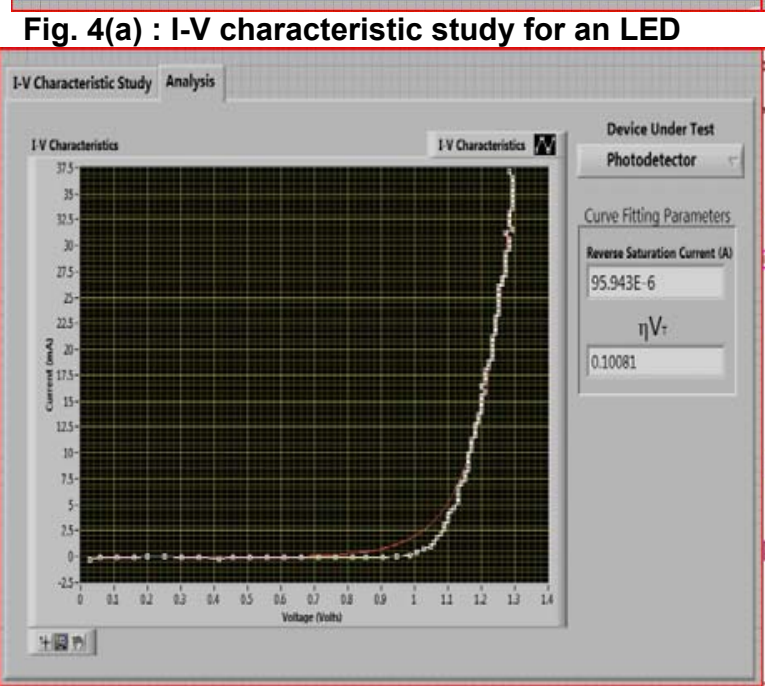

Fig. 4(c): I-V Characteristic of Photodetector
Fig. 4(b): I-V Characteristic study for Laser Diode

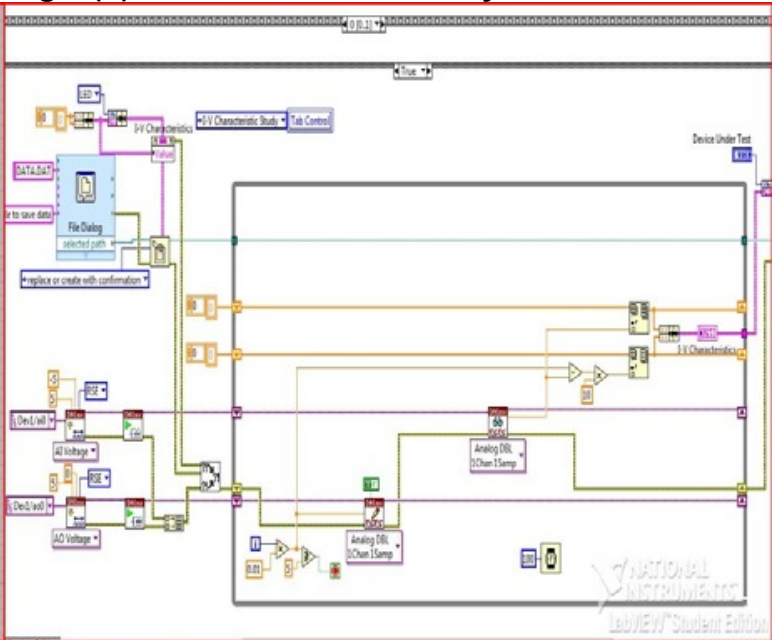

Fig. 5 : Part of software code for I-V characteristics

2. P-I Characteristics : The experimental set up for P-I characteristic study is shown in Fig. 6. 


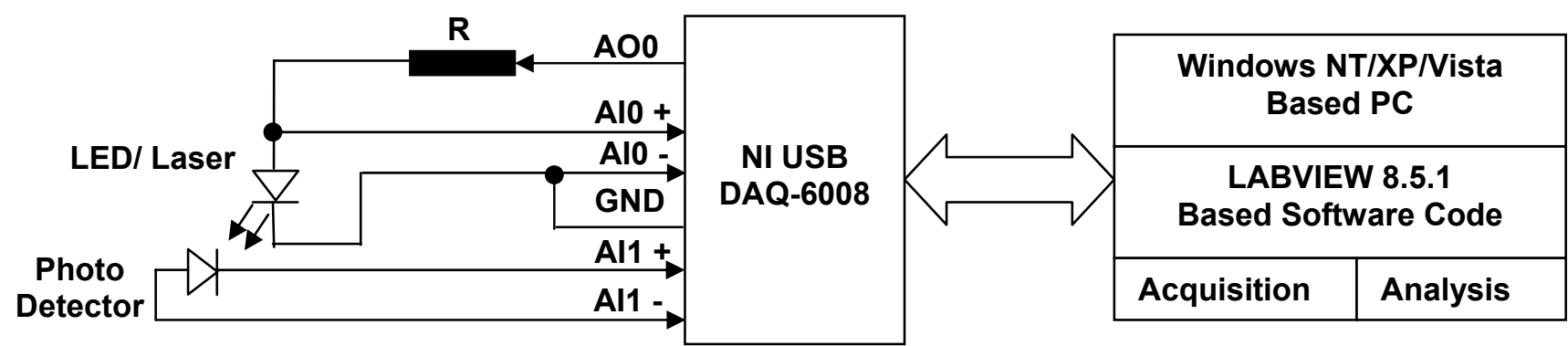

Fig. 6 : Experimental set-up for P-I characteristics study

The $P$-I characteristics of an LED/Laser diode is obtained by varying the current flowing through the device using Analog Output Channel 0 (AO0) for the DAQ card. The current in the device is measured by acquiring the device voltage at Analog Input Channel 0 (AIO) in differential mode. The light emitted by the device is detected by the photo detector aligned in front of the LED/laser diode. The voltage developed at the output of the detector corresponds to the optical power output of the device under study. This voltage is sent to the developed module at Analog Channel 1 of the DAQ card in differential mode. This voltage is calibrated in terms of optical power at the beginning of routine. The calibration of the detector may be done either in online or offline mode. The online mode helps the user to calibrate an unknown detector with a standard light source before acquisition of P-I characteristics. When working in offline mode, the calibration factor calculated earlier can be loaded from a datalog file for all calculations and acquisition of characteristics.

The P-I characteristics of an LED and a laser diode are shown in Fig. 7(a)-(b). The analysis of the results predicts an increase in optical power for an LED with current. However, the light emission is spontaneous in case of an LED as compared to a Laser diode which exhibits similar P-I characteristics for current values below threshold. Above this current, the output optical power of a laser increases linearly thus verifying the stimulated emission in this region.

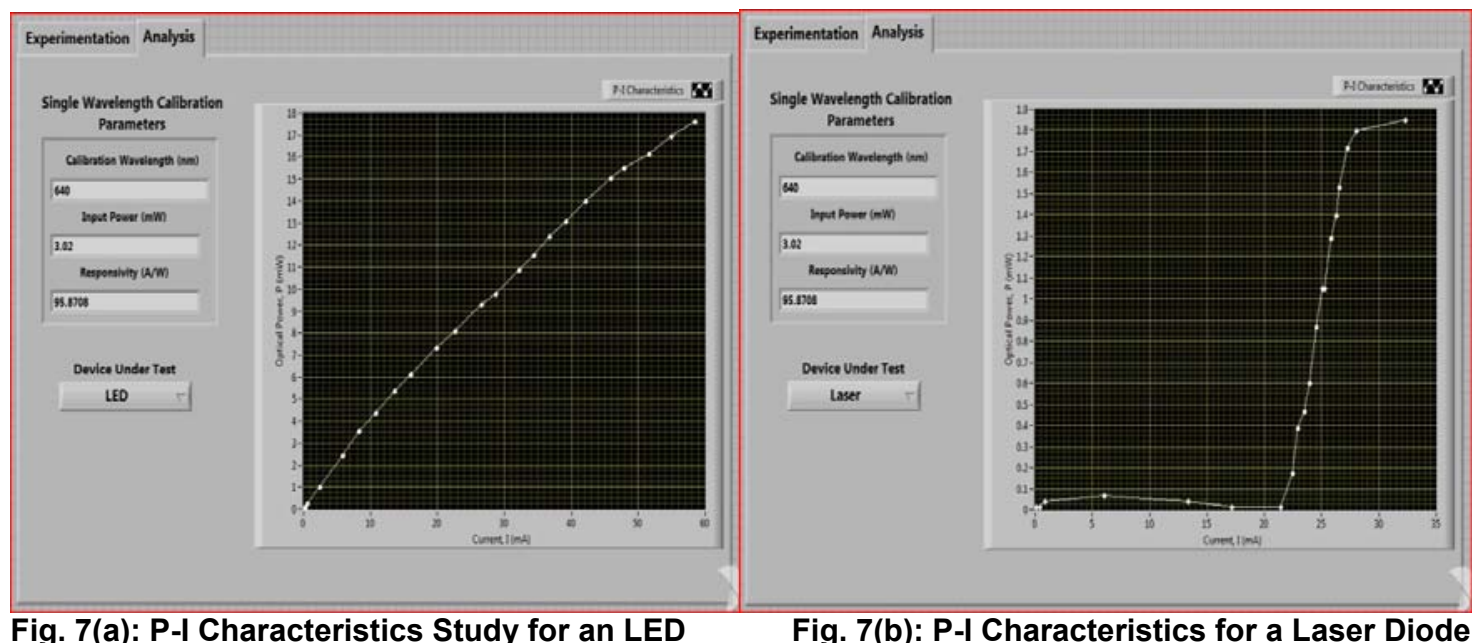

The software code for the study of P-I characteristics is illustrated in Fig. 8. The software converts the photo detector output to its corresponding optical power using its calibration factor. This optical power is plotted with respect to device current and simultaneously stored in a datalog file for future analysis and study. 


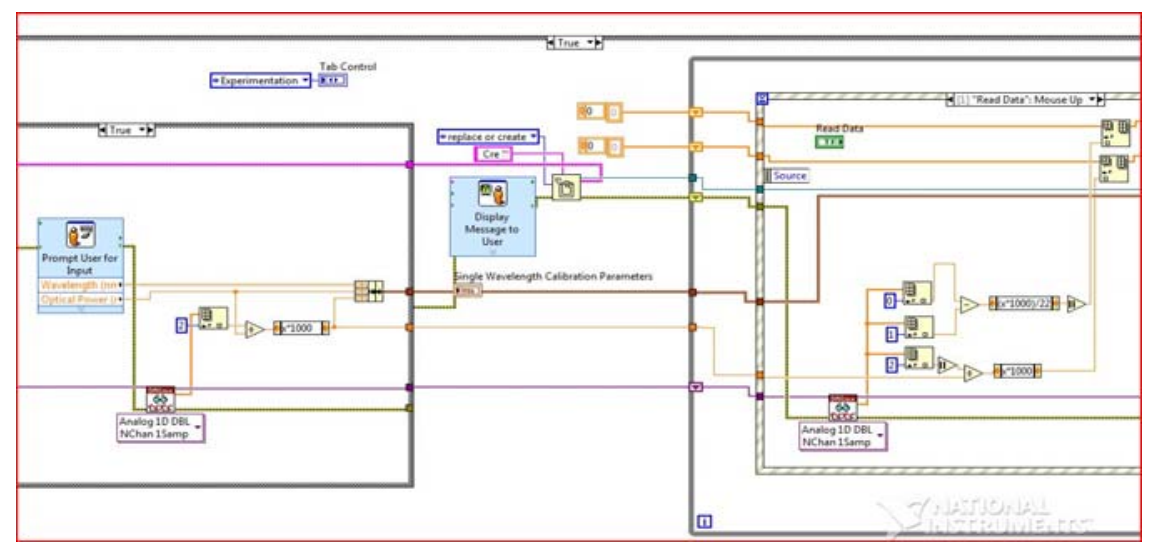

Fig. 8: Part of the graphical software code for study of P-I characteristics

Through the analysis of LEDs and laser diode, the difference between the light emission processes in two devices can clearly be shown. The results obtained suggest that luminous intensity in spontaneous region for the laser diode is less than that of the light emitting diode. This effect is well explained by the fact that for lower forward bias current, the number of holes and electrons injected is small. As a result, the gain in the device too small to overcome the cavity loss.

Also the region after the threshold current value for laser diode follows a smooth curve without any kinks or abruptness in the characteristics. The differential slope efficiency for this region is constant at all points in the curve. Both these results indicate that the quantum efficiency of the laser diode used is excellent and the internal defects in the laser are minimum.

\section{SECTION III : CCD Based Optical Experiments}

When a laser beam passes through a pin hole, the distribution of light shows a bright maxima surrounded by a number of secondary minima of decreasing intensity. The diffraction pattern is in the form of concentric circles where there are bright areas between the dark concentric rings. The phenomenon of diffraction can be studied under two heads, namely Fresnel and Fraunhoffer diffraction. Fraunhoffer diffraction is a special case of Fresnel diffraction when the source illuminating the aperture and the observation screen is located at infinity. When a lens is placed after the aperture, the diffraction pattern studied at the back of its focal plane, the diffraction is called as Fraunhoffer diffraction. We study the Fresnel diffraction in the present experiment. ${ }^{[7],[8]}$

\section{Theory}

The working formula for calculating the diameter of the pin hole is as follows. Let the diameter of the pin hole be $d$ and the screen is placed at a distance $b$ from the pin hole, then the radius of the nth dark ring is given as

$$
x_{n}=\frac{n b \lambda}{d}
$$

When the laser light falls on the pin hole, one can see the appearance of many concentric circles. There are bright areas separating the dark concentric rings. The dark ring at the centre of the diffraction pattern is the central maxima and adjacent to it are different orders. The distance between the central maxima and the first order maxima can be used to calculate pin hole diameter using the equation (4)

$$
\mathrm{d}=\frac{\mathrm{nb} *}{\mathrm{x}_{\mathrm{n}}}
$$

\section{Experimental Set-up}

The experimental set up for the CCD based pin hole Fresnel diffraction study is shown in Fig. 9 . 


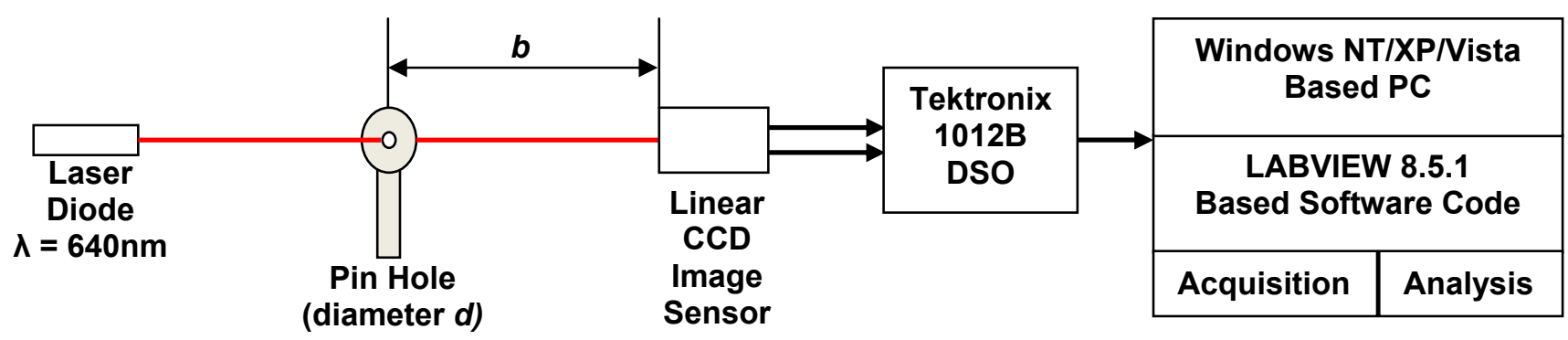

Fig. 9 : Experimental Set-up for Single Slit Diffraction Study

Fig. 9 describes the experimental set-up used for pin hole diffraction studies using a linear CCD sensor. The diffraction pattern is generated when a beam of laser passes through the pin hole. This pattern is made to fall on a linear CCD image sensor which has 1024 elements with inter-pixel separation of $7.8 \mu \mathrm{m}$. Thus, the total length of the array is $7.9872 \mathrm{~mm}$. which corresponds to $5.2 \mathrm{~ms}$ on the time frame of an oscilloscope. Each pixel data is then acquired by a LabVIEW based graphical code through Textronix 1012B Digital Storage Oscilloscope (DSO). The analysis of this data reveals a diffraction pattern exhibiting maxima and minima points within the pattern which is stored in a text file simultaneously for further analysis. The spacing between two maxima or minima can easily be computed by the placing the cursor at the desired points and pressing the "Calculate Distance" button on the software routine as shown in Fig. 10(a) and 10(b). These distances are stored in an array and displayed on front panel of the software every time the distance is calculated by the user.

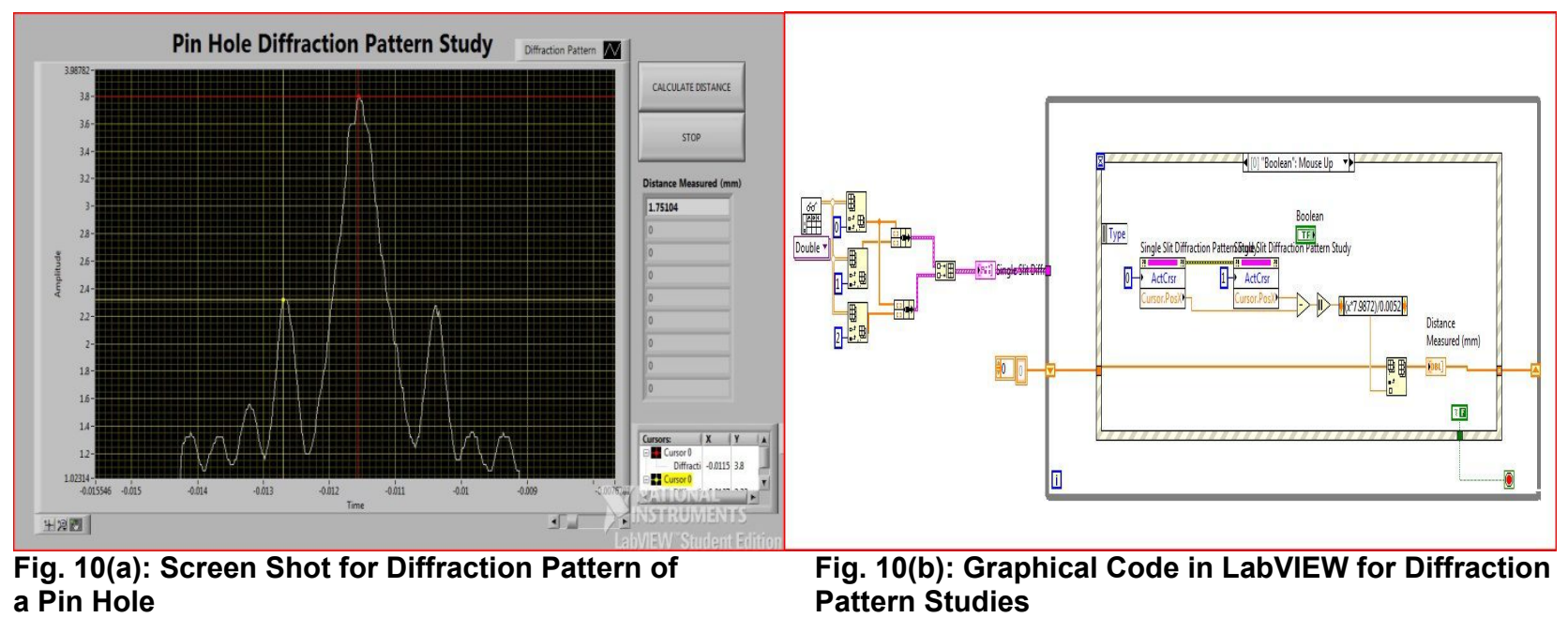

The distance between the central and the first order maxima as calculated from the fringe pattern is further used to calculate the pin hole diameter. The calculated value from the experimental data for a pin hole of $175 \mu \mathrm{m}$ is $170 \mu \mathrm{m}$.

The diffraction pattern obtained using CCD is in accordance with the theoretical patterns. The diffraction patterns obtained help the students understand the variation in light patterns with ease. In the conventional optics laboratory, students calculate the distance between two maxima points on the concentric ring pattern for diameter calculations. However, the actual pattern shape is never visible which makes it difficult to relate the results with theory and to understand the basic concept of diffraction. Thus the developed automated 
system is an improvement over earlier experiments carried out in the laboratory along with increased accuracy and reliability in results.

\section{SECTION IV : Advanced Applications in Optics}

Applied optics is the application of the optics to the broad realm of practical problems in industry, engineering and science. The invention of optical devices like the laser, optical fibers and solid state detectors has led to a wide range of new technologies. The laboratory developed aims at using optics for multidisciplinary studies like Lambert Beers law study in chemisty, heart rate monitoring and jaundice studies in the medical field.

This section describes one of the applications of optics in the area of chemical education by studying Lambert Beers law. In chemistry, the Lambert-Beer law is an empirical relationship that relates the absorption of light to the properties of the material through which the light is travelling. Beers law can be applied to the analysis of a mixture by spectrophotometry, without the need for extensive pre-processing of the sample. An example is the determination of biliburin in blood plasma samples. The law is widely used in infra-red spectroscopy for analysis of polymer degradation and oxidation. This law also describes the attenuation of solar or stellar radiation as it travels through the atmosphere. ${ }^{[9]}$

\section{Theory}

Substances that appear to have color absorb light at wavelengths that are visible. The color perceived is due to the wavelengths that are not absorbed. It is the light that is either transmitted or reflected that is sensed by the eye. Fig. 11 shows a beam of monochromatic radiation of radiant power $\mathrm{P}_{0}$ directed at a sample solution. Absorption takes place and the beam of radiation leaving the sample has radiant power $P$.

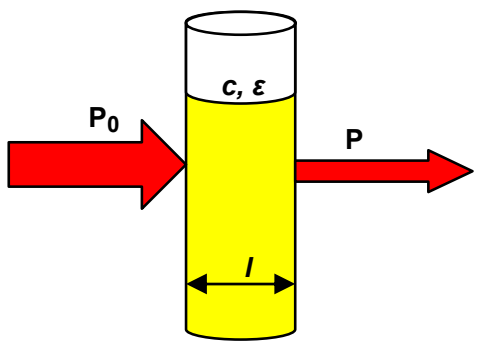

Fig. 11: Light Absorption in a diluted solution

The amount of radiation absorbed defined as absorbance, A which may be measured using equation (5).

$A=\log _{10}\left(P_{0} / P\right)$

Lambert Beers law states that the absorbance, A of a dissolved substance is a linear function of its concentration, c. The distance the light travels through the material, i.e., the path length, $l$, and the molar absorbivity, $\varepsilon$, determine the slope of the linear plot as given by the equation (6).

$A=$ ale

The Lambert-Beers law is valid only for diluted solutions. The limits for its validity differ for different materials. As a general rule, every material showing absorption of up to 0.5-0.6 still obeys the Lambert-Beer Law.

In the developed set up, we have reported the behavior of the linear absorption increase of Nickel Sulphate $\left(\mathrm{NiSO}_{4}\right)$ with increasing concentration in order to determine its molar absorbivity at a given wavelength within its absorption area. The studies have been carried out for various solutions.

\section{Experimental Set-up}

Fig.12 shows the experimental set up for Lambert Beers Law study. A light beam of red laser with wavelength of $640 \mathrm{~nm}$ was made to pass through a cuvette containing a solution of nickel sulphate. The amount of light 
transmitted out of the solution was made to fall on a photodetector and its output voltage was acquired using an Analog Input Channel AIO of the DAQ card in differential mode through LabVIEW.

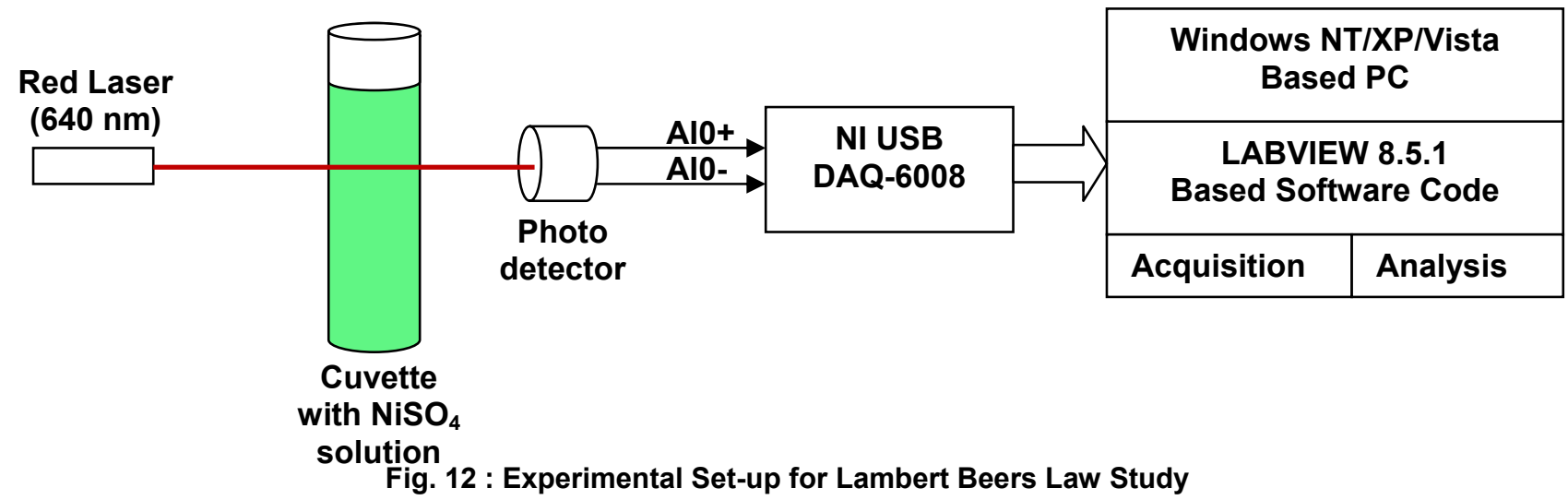

The process was repeated for solutions of different concentrations and the data stored in a datalog file. The path length for the cuvette was also stored in the data file for absorbivity calculations. The photo detector output was converted into the corresponding optical power and the absorbance was calculated for all set of concentrations. The absorbance vs. concentration curve was simultaneously plotted for each concentration value of the solution and fitted linearly as depicted in fig. 13(a)-(b)

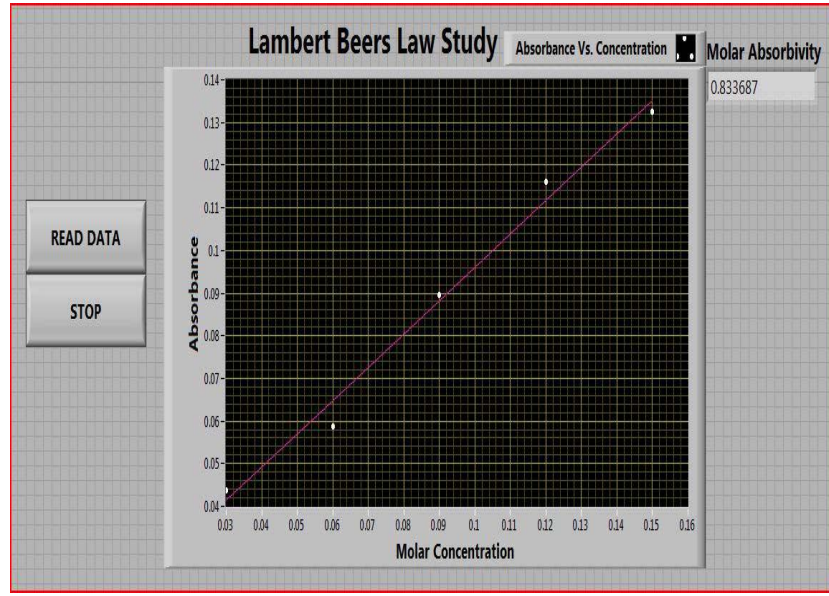

Fig. 13(a) : Screen-shot for Lambert-Beers Law study module

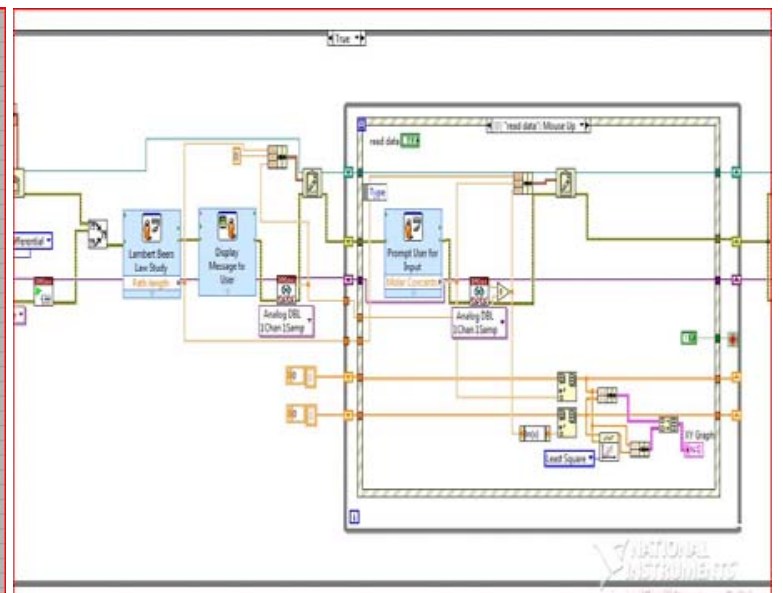

Fig. 13(b) : Screen shot for part of the developed software code for Lambert Beers Law study

The linear variation of the plot verified the Lambert Beers Law. The data points were curve fitted linearly and the average absorbivity calculated for different solutions.

\section{CONCLUSIONS}

1. The developed modern automated optics laboratory is an improvement over the conventional optics laboratory. The experiments which could not be performed earlier like diffraction pattern studies can now performed with ease and accuracy. Also, the results obtained with the automated system are more accurate and precise.

2. The modern optics laboratory is being envisaged to meet higher goals and the work is in progress for developing new experiments like heart rate monitoring system, non-evasive jaundice studies from the skin and advanced interference, diffraction and polarization studies. The development of optical 
instruments like CCD based Spectrometer and integrated Optical Energy-Power Meter is also under execution.

3. The developed laboratory will invoke student interest by exposing them to various modern tools in comparison to very conventional as well as boring existing optics laboratories. The automation of the experiments will also save great amount of experimentation time and procurement of costly equipments dedicated to each experiment thus providing an efficient way to carry out studies with reduced financial constraints and better manoeuvrability.

4. The use of the scientific graphical programming software helps in performing various real time measurements and calculations with ease.

\section{ACKNOWLEDGEMENTS}

Authors, Amit Garg and Vishal Dhingra, duly acknowledge University Grants Commission, New Delhi for providing the financial assistance under the major research proposal scheme for the work reported in this paper against sanction no 34-62/2008(SR) for the project entitled "Investigating science hands-on to promote innovation and research at the undergraduate level".

\section{REFERENCE CITATIONS}

[1] Mallalieu K., Arieatas R., Soapos;Brien D., "An inexpensive PC-based laboratory configuration for teaching electronic instrumentation", IEEE Transactions on Education, Volume 37, Issue 1, pp. 91 - 96 (1994)

[2] Boreman G. D., "Teaching optical engineering to electrical-engineering undergraduates", SPIE Vol. 3190, pp. 205-207 (1997)

[3] Garg A., Sharma R., Dhingra V., Ali N., Gambhir R., "Development of a PC based Optical Power Meter", Proceedings of International Conference on Trends in Optics and Photonics, pp.: 406-413 (2009).

[4] E. Redondo, A. Ojeda, G. Gonzalez Dı'az, I. Ma'rtil, "A laboratory experiment with blue light-emitting diodes", Am. J. Phys. 65(5), pp. 371-376 (1997)

[5] A M Ojeda, E Redondo, G Gonz'alez D'ıaz, I M'artil, "Analysis of light-emission processes in lightemitting diodes and semiconductor lasers", Eur. J. Phys. 18, pp. 63-67 (1997).

[6] Dwivedi A., Ali N., Bisht D., Saxena N., Garg A., Sharma R., Dhingra V., "Understanding L-I Characteristics of Light Emitting Diodes and Laser Diodes", Proceedings of The Second National Conference on Mathematical Techniques: Emerging Paradigms for Electronics and IT Industries (MATEIT-2008)

[7] Elliott K.H., Mayhew C.A., "The use of commercial CCD cameras as linear detectors in the physics undergraduate teaching laboratory", Eur. J. Phys. 19, pp. 107-117 (1998)

[8] Ramil A., Lopez A.J., Vincitorio F., "Improvements in the analysis of diffraction phenomenon by means of digital images", American Journal of Physics, Vol. 75, Issue 11, pp. 999-1002 (2007).

[9] Mathpal S., Kandpal N.D., "Colorimetric Estimation of Ni(II) lons in Aqueous Solution", E-Journal of Chemistry, Vol. 6(2), pp. 445-448 (2009) 